IUCrJ

ISSN 2052-2525

BIOLOGY|MEDICINE

Received 16 February 2018

Accepted 4 June 2018

Edited by Z.-J. Liu, Chinese Academy of Sciences, China

Keywords: catalysis; redox biology; structural biology; enzyme mechanism; denitrification; nitrogen cycle; copper-haem nitrite reductases; Ralstonia pickettii.

PDB references: $R p N i R$ D97N-NO $\mathrm{N}_{2}^{-}, 50 b o$; RpNiR D97N-NO, 5ocb; wt RpNiR-NO, 5ocf; T2D RpNiR, 6fja; wt RpNiR, 6f1q

Supporting information: this article has supporting information at www.iucrj.org

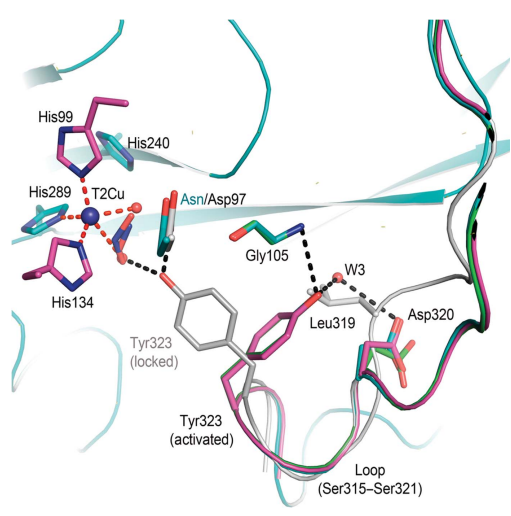

OPEN $\odot$ ACCESS

\section{Identification of a tyrosine switch in copper-haem nitrite reductases}

\author{
Jianshu Dong, Daisuke Sasaki, Robert R. Eady, Svetlana V. Antonyuk and S. Samar \\ Hasnain*
}

Molecular Biophysics Group, Institute of Integrative Biology, Faculty of Health and Life Sciences, University of Liverpool, Liverpool L69 7ZX, England. *Correspondence e-mail: s.s.hasnain@liverpool.ac.uk

There are few cases where tyrosine has been shown to be involved in catalysis or the control of catalysis despite its ability to carry out chemistry at much higher potentials ( $1 \mathrm{~V}$ versus $\mathrm{NHE})$. Here, it is shown that a tyrosine that blocks the hydrophobic substrate-entry channel in copper-haem nitrite reductases can be activated like a switch by the treatment of crystals of Ralstonia pickettii nitrite reductase $(R p \mathrm{NiR})$ with nitric oxide $(\mathrm{NO})(-0.8 \pm 0.2 \mathrm{~V})$. Treatment with NO results in an opening of the channel originating from the rotation of Tyr323 away from $A_{\text {CAT }}$ 97. Remarkably, the structure of a catalytic copper-deficient enzyme also shows Tyr323 in the closed position despite the absence of type 2 copper $(\mathrm{T} 2 \mathrm{Cu})$, clearly demonstrating that the status of Tyr323 is not controlled by $\mathrm{T} 2 \mathrm{Cu}$ or its redox chemistry. It is also shown that the activation by NO is not through binding to haem. It is proposed that activation of the Tyr323 switch is controlled by NO through proton abstraction from tyrosine and the formation of HNO. The insight gained here for the use of tyrosine as a switch in catalysis has wider implications for catalysis in biology.

\section{Significance}

Structural characterization of the copper-haem nitrite reductase (haem $\mathrm{CuNiR})$ from Ralstonia pickettii $(R p \mathrm{NiR})$ revealed a tyrosine residue blocking the substrate-entry channel and binding site. The treatment of crystals with NO triggers a movement of tyrosine that allows NO- and $\mathrm{NO}_{2}^{-}$-bound species to be captured to provide the first information on ligand-bound species in this class of nitrite reductases. The use of tyrosine as a switch in activating the redox enzyme $R p N i R$ may have wider significance as this tyrosine is found to be totally conserved in all known haem CuNiRs.

\section{Introduction}

In biology, redox reactions and catalysis are often performed by redox metals and their cofactors, which typically possess potentials of less than $400 \mathrm{mV}$. The transition metals iron, copper and manganese are the most utilized redox centres in biology, either on their own or as a component of cofactors such as iron-sulfur clusters, copper-sulfur clusters or haem (Liu et al., 2014). In some cases redox centres are coupled to orchestrate the delivery of protons and electrons to the catalytic centre for substrate reduction. An example of such an inter-linked mechanism in which the delivery of electrons, substrate and protons are well controlled and regulated through coupled potentials is the well studied copper nitrite reductases $(\mathrm{CuNiRs})$ that catalyse the reaction $\mathrm{NO}_{2}^{-}+\mathrm{e}^{-}$ $+2 \mathrm{H}^{+} \leftrightarrow \mathrm{NO}+\mathrm{H}_{2} \mathrm{O}$, a step in the microbial ATP-generating denitrification pathway (Maia \& Moura, 2014). The extensive 
application of nitrogeneous fertilizers has resulted in agriculture being the largest source of atmospheric $\mathrm{N}_{2} \mathrm{O}$. Denitrifying microorganisms that utilize nitrate as a terminal respiratory electron acceptor produce this potent ozonedepleting and greenhouse gas. Copper-containing nitrite reductase $(\mathrm{CuNiR})$ is a key enzyme in this process since it forms $\mathrm{NO}$, a precursor for $\mathrm{N}_{2} \mathrm{O}$ formation, as the product of the one-electron reduction of nitrite. Electron transfer from a partner cupredoxin or cytochrome redox protein to $\mathrm{CuNiR}$ provides the electrons for this reduction.

Structural studies of many CuNiRs have shown that a duplicated cupredoxin-domain monomer unit forms the core of the trimeric enzymes (designated here as two-domain CuNiRs). They contain two types of metal centre: a type $1 \mathrm{Cu}$ $(\mathrm{T} 1 \mathrm{Cu})$ site that accepts electrons from a physiological donor and a catalytic type $2 \mathrm{Cu}(\mathrm{T} 2 \mathrm{Cu})$ centre with $\mathrm{His}_{3}-\mathrm{H}_{2} \mathrm{O}$ ligation. The two centres are separated by a $\sim 12.6 \AA$ Cys-His bridge which functions in proton-gated electron transfer (Godden et al., 1991; Boulanger \& Murphy, 2002; Ellis et al., 2003; Tocheva et al., 2004; Antonyuk et al., 2005; Lawton et al., 2013). The active-site pocket has an aspartic acid and a histidine residue that are conserved in all $\mathrm{CuNiRs}$ and that mutational studies have shown to be essential for effective catalysis (Kataoka et al., 2000; Boulanger et al., 2000; Prudêncio et al., 2001; Ellis et al., 2002). These residues, designated $\mathrm{Asp}_{\mathrm{CAT}}$ and $\mathrm{His}_{\mathrm{CAT}}$, are linked to the catalytic centre via a water bridge. The binding of nitrite at the $\mathrm{T} 2 \mathrm{Cu}$ centre displaces the $\mathrm{H}_{2} \mathrm{O}$ ligand, induces subtle changes to these residues and significantly increases the reduction potential so as to promote electron transfer from the $\mathrm{T} 1 \mathrm{Cu}$ centre, gated by protonation of Asp $\mathrm{P}_{\mathrm{CAT}}$ (Hough, Antonyuk et al., 2008; Brenner et al., 2009; Ghosh et al., 2009; Leferink et al., 2011). This orchestrated sequence of events minimizes the potential for the formation of a deactivated species with a prematurely reduced $\mathrm{T} 2 \mathrm{Cu}$ site from which the solventderived water ligand dissociates before nitrite can bind.

Two putative proton-pathway channels at the monomer interface that lead to the $\mathrm{T} 2 \mathrm{Cu}$ catalytic site have been identified in two-domain CuNiRs and one has been established to be the substrate-access channel from bulk solvent (Ellis et al., 2003; Antonyuk et al., 2005). This channel, which is approximately $6 \AA$ wide, is hydrophobic and is formed by residues from two adjacent monomers. The architecture of this channel has been shown to be important in controlling the coordination geometry of bound nitrite as $\eta^{2}-O, O$ or end-on $\eta^{1}-O$ (Antonyuk et al., 2005; Fukuda et al., 2014; Boulanger \& Murphy, 2003) and in determining the rate-limiting step in turnover (Leferink et al., 2014).

Two new subclasses of CuNiRs have been identified that retain the core structure of the two-domain enzymes but have an extra cupredoxin or cytochrome $c$-containing domain fused at the amino- or carboxy-terminus, respectively. Although they have only recently been recognized, genome analysis shows that both of these classes are widely distributed among Gram-negative $\alpha$ - and $\gamma$-proteobacteria isolated from a range of different habitats (Bertini et al., 2006; Ellis et al., 2007; Antonyuk et al., 2015). The first structurally characterized three-domain haem $\mathrm{CuNiR}$, that from Ralstonia pickettii (RpNiR; Han et al., 2012; Antonyuk et al., 2013), is trimeric, with the haem $c$ domain of one monomer in close proximity to the $\mathrm{T} 1 \mathrm{Cu}$ site of another monomer that is well placed for effective electron transfer, with a haem- $\mathrm{T} 1 \mathrm{Cu}$ separation of 10.6 $\mathrm{A}$. RpNiR, with its additional tethered cytochromecontaining domain, provides a naturally fused electrontransfer complex, providing an opportunity to compare the roles of specific amino-acid residues in inter-domain electron transfer with transient protein complexes. Comparison of the structure with the binary complex $A x \mathrm{NiR}$-cytochrome $c_{551}$ and mutagenesis studies have provided direct evidence for the importance of a hydrogen-bonded water at the haemcupredoxin domain interface in haem- $\mathrm{T} 1 \mathrm{Cu}$ electron transfer, in contrast to the $\mathrm{C}-\mathrm{C}$ interactions of the binary complex (Antonyuk et al., 2013; Nojiri et al., 2009).

The structure of the related three-domain haem-CuNiR from Pseudoalteromonas haloplanktis $(P h \mathrm{NiR})$ showed differences in the overall organization of the subunits (Tsuda et al., 2013). In PhNiR the linker wraps around the neighbouring monomer to reach the distant third monomer to create the cytochrome-catalytic domain interface with an extensive water network as in $R p \mathrm{NiR}$.

Surprisingly, given the retention of the catalytic core architecture of the two-domain NiRs, in both $R p N i R$ and $P h \mathrm{NiR}$, the hydrophobic substrate-access channel is blocked by Tyr323 (Antonyuk et al., 2013; Tyr313 in PhNiR; Tsuda et al., 2013), a residue that forms part of the linker between the cytochrome $c$ and cupredoxin domains. It had remained a puzzle how the substrate reaches the catalytic site and it has been speculated that the nonfunctional proton channel of the two-domain NiRs might also be used for substrate delivery (Antonyuk et al., 2005).

\section{Methods}

2.1. Cloning, expression, purification, crystallization and structure determination

Site-directed mutagenesis was performed using the QuikChange site-directed mutagenesis kit (Agilent). The primers were $R p \mathrm{NiR}$ D97N, sense 5'-GCCGCACAACATCACCTG CACGGCGT-3' and antisense 5'-ACGCCGTGCAGGTTAT GTTGTGCGGC-3'. The mutations were confirmed by sequencing before transformation. $R p \mathrm{NiR}$ and the $R p \mathrm{NiR}$ D97N mutant were expressed in Escherichia coli BL21(DE3) cells and purified and assayed as described previously (Han et $a l ., 2012)$. For T2D $R p N i R$ the copper-incorporation stage of purification was omitted, resulting in the absence of copper from the $\mathrm{T} 2 \mathrm{Cu}$ site. Crystals appeared in $2-3$ weeks at $4^{\circ} \mathrm{C}$. $R p \mathrm{NiR}-\mathrm{NO}$ complexes were obtained by treating crystals with NO. An $R p$ NiR D $97 \mathrm{~N}-\mathrm{NO}$ crystal was incubated in $100 \mathrm{mM}$ sodium nitrite in reservoir solution to obtain the $R p \mathrm{NiR}$ D97N- $\mathrm{NO}_{2}^{-}$complex. The crystals were cryoprotected in the reservoir solution with $10 \%$ glycerol and flash-cooled in liquid nitrogen. Diffraction data were collected from single crystals at $100 \mathrm{~K}$ on the I04, I04-1 and I02 PX beamlines at Diamond 
Table 1

Data-collection and refinement statistics.

Values in parentheses are for the highest resolution shell.

\begin{tabular}{|c|c|c|c|c|c|}
\hline & $R p \mathrm{NiR} \mathrm{D} 97 \mathrm{~N}-\mathrm{NO}_{2}^{-}$ & $R p \mathrm{NiR}$ D97N-NO & Wt $R p N i R-N O$ & $\mathrm{~T} 2 \mathrm{D} R p \mathrm{NiR}$ & Wt $R p N i R$ \\
\hline DLS beamline & I02 & I02 & I04-1 & I04 & I03 \\
\hline Space group & $I 2_{1} 3$ & $I 2_{1} 3$ & $I 2_{1} 3$ & $H 3$ & $I 2_{1} 3$ \\
\hline$R_{\text {merge }} \dagger$ & $0.12(0.85)$ & $0.09(0.65)$ & $0.13(0.96)$ & $0.08(0.45)$ & $0.11(0.73)$ \\
\hline$\langle I / \sigma(I)\rangle$ & $6.7(1.5)$ & $22(1.7)$ & $9.7(1.4)$ & $9.2(2.1)$ & $13.5(2.1)$ \\
\hline Completeness (\%) & $99.2(99.2)$ & $98.4(99.4)$ & $100(99.8)$ & $98.9(90.9)$ & $99.6(99.2)$ \\
\hline No. of reflections & 74954 & 88395 & 92042 & 86243 & 41187 \\
\hline$R_{\mathrm{work}} / R_{\text {free }} \neq$ & $0.147 / 0.175$ & $0.143 / 0.161$ & $0.149 / 0.164$ & $0.136 / 0.17$ & $0.134 / 0.161$ \\
\hline \multicolumn{6}{|l|}{ No. of atoms } \\
\hline Protein & 3677 & 3660 & 3694 & 3479 & 3535 \\
\hline Water & 643 & 790 & 681 & 437 & 442 \\
\hline \multicolumn{6}{|l|}{$B$ factors $\left(\AA^{2}\right)$} \\
\hline PDB code & 5obo & 5 ocb & 5 ocf & 6 fja & $6 \mathrm{f} 1 \mathrm{q}$ \\
\hline
\end{tabular}

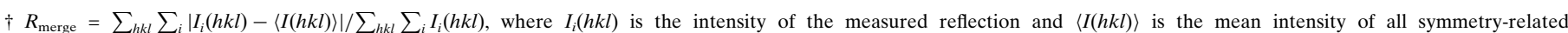

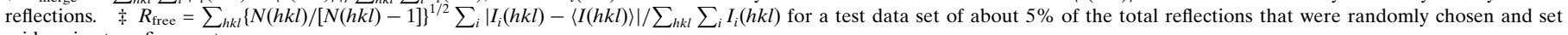
aside prior to refinement.

Light Source. X-ray data were processed with $X D S$ (Kabsch, 2010) for the T2D $R p \mathrm{NiR}$ and wild-type (wt) $R p \mathrm{NiR}$ structures and MOSFLM (Battye et al., 2011) for all other structures and were merged by AIMLESS (Evans \& Murshudov, 2013 ) in the CCP4 program suite (Winn et al., 2011). The $R p \mathrm{NiR}$ D97N-NO structure was solved by molecular replacement using PDB entry 3ziy (Antonyuk et al., 2013) as the search model, refined using REFMAC5 (Murshudov et al., 2011) and rebuilt in Coot (Emsley et al., 2010). The $R p \mathrm{NiR}$ D97N-NO and wt $R p$ NiR-NO structures were isomorphous to the $R p \mathrm{NiR}$ structure which was used as the starting model for the refinement of both structures. Water molecules and ligands were added manually in Coot. $\mathrm{H}$ atoms were added at riding positions at the end of refinement. The quality of the model was assessed using MolProbity (Chen et al., 2010). Data-collection and refinement statistics are summarized in Table 1. Channels, tunnels and pores were visualized using MOLE (Sehnal et al., 2013). Structural figures were prepared using PyMOL (v.1.8; Schrödinger).

\subsection{Activity assay}

The nitrite reductase activities of $R p \mathrm{NiR}$ and the $R p \mathrm{NiR}$ D97N mutant were measured using an NO electrode with ascorbate-reduced phenazine methosulfate as the electron donors in a glovebox under a nitrogen atmosphere, as described previously (Han et al., 2012).

\section{Results}

Mutagenesis and structural studies combined with computational analysis of several two-domain CuNiRs have estab- lished a role for the invariant $\mathrm{Asp}_{\mathrm{CAT}}$ residue in the catalytic pocket in proton donation to bound nitrite and in promoting electron transfer from $\mathrm{T} 1 \mathrm{Cu}$ to the $\mathrm{T} 2 \mathrm{Cu}$ site (Brenner et al., 2009; Ghosh et al., 2009; Leferink et al., 2011). Comparative analysis of the peptide sequences of cytochrome-fused and cupredoxin-fused three-domain NiRs showed that the corresponding aspartic acid residue was conserved (Supplementary Fig. S1), suggesting a similar role for Asp97 of $R p N i R$ in catalysis. We constructed and purified the $R p \mathrm{NiR} D 97 \mathrm{~N}$ variant enzyme. The normal incorporation of iron and copper and the proper reconstitution of the catalytic $\mathrm{T} 2 \mathrm{Cu}$ site were evident from an anomalous diffraction map of the enzyme crystals (Fig. 1e) and spectroscopic analysis. The electron paramagnetic resonance (EPR) spectrum of the D97N mutant was found to be identical to that of wt $R p \mathrm{NiR}$, showing that both the $\mathrm{T} 1 \mathrm{Cu}$ and $\mathrm{T} 2 \mathrm{Cu}$ centres were oxidized. As for wt $R p \mathrm{NiR}$, the EPR spectrum remained invariant with nitrite, indicating a lack of binding to the catalytic site or its immediate surroundings. The $R p \mathrm{NiR} D 97 \mathrm{~N}$ variant enzyme was found to be inactive, as expected owing to impaired proton delivery to the active site.

Despite the fact that the structures of two different haem CuNiRs were published five years ago (Antonyuk et al., 2013; Tsuda et al., 2013), no nitrite-bound structures have been reported. Our own efforts to obtain nitrite-bound structures with both wt $R p \mathrm{NiR}$ and the $\mathrm{D} 97 \mathrm{~N}$ mutant failed. In the course of experiments to test whether the nonfunctional proton channel of the two-domain NiRs might also be used for substrate delivery, we made the unexpected observation that the pre-exposure of crystals of wt $R p \mathrm{NiR}$ to NO, which has a potential of $-0.8 \mathrm{~V}$, allowed structure determination of 
the NO-bound enzyme. NO treatment results in the activation of Tyr323 such that the hydrogen bond to $\mathrm{Asp}_{\mathrm{CAT}} 97$ is broken, freeing Tyr323 to move away from the substrate-binding pocket and resulting in opening of the substrateaccess channel. Similar treatment of the active-site Asp CAT $_{\text {T }}$ D97N mutant enzyme in which proton donation to the bound substrate is impaired additionally allowed the first structural determination of a nitrite-bound species of a haem CuNiR.

\subsection{Structures of wt $R p N i R-N O$ and $R p N i R$ D $97 N-N O$}

The structure of as-isolated $R p \mathrm{NiR} \mathrm{D} 97 \mathrm{~N}$ was very similar to that of the wild-type enzyme, including the locked-down position of Tyr323. Exposure of the crystals to NO enabled the structures of NO-bound $R p \mathrm{NiR} \mathrm{D} 97 \mathrm{~N}$ and wt $R p \mathrm{NiR}$ to be determined at around $1.8 \AA$ resolution (Figs. $1 a$ and $1 b$ ). Refinement confirmed that $\mathrm{NO}$ was bound to $\mathrm{T} 2 \mathrm{Cu}$ in an asymmetric side-on manner with distances of the $\mathrm{N}_{\mathrm{NO}}$ and $\mathrm{O}_{\mathrm{NO}}$ atoms to copper of $\sim 2.0$ and $\sim 2.6 \AA$ in wt $R p \mathrm{NiR}$ and $\sim 2.0$ and $2.8 \AA$ in the mutant structure, respectively. The proximity of NO to the side chain of Asp97/Asn97 (Figs. $1 a$ and $1 b$ ) indicated a probable hydrogen bond (Asp97 $\mathrm{O}^{\delta 2} /$ Asn97 $\mathrm{N}^{\delta 2}$ to $\mathrm{N}_{\mathrm{NO}}$ distance of $3.1 \AA$ ). In contrast, NO interacts only weakly if at all with His 240 , with distances of about $3.5 \AA$ for wt $R p \mathrm{NiR}$ and $3.6 \AA$ for $R p \mathrm{NiR} \mathrm{D} 97 \mathrm{~N}$. The binding of NO results in a $90^{\circ}$ flip of the Tyr323 side chain, disrupting a hydrogen bond to $\operatorname{Asp}_{\mathrm{CAT}} 97$ to form a new hydrogen bond to Gly105 N (Figs. $2 a$ and $2 b$ ). This is accompanied by a large movement of the linker loop, Ser315-Ser321, that connects the haem and cupredoxin domains, resulting in the opening of the blocked channel from bulk solvent to the $\mathrm{T} 2 \mathrm{Cu}$ site. As these structures are from a crystal that grew in space group $I 2{ }_{1} 3$, differing from our previously reported structures of wt $R p \mathrm{NiR}$ ( $H 3$ and $P 2_{1} 3$ ) and mutants $(H 3)$, the structure of as-isolated wt $R p \mathrm{NiR}$ was also determined in space group $I 2{ }_{1} 3$ at $2.3 \AA$ resolution (Fig. 1c). A comparison of this and all other structures of untreated crystals, including that from another haem CuNiR, PhNiR, shows the invariance of the tyrosine position irrespective of the space group or enzyme. In all cases

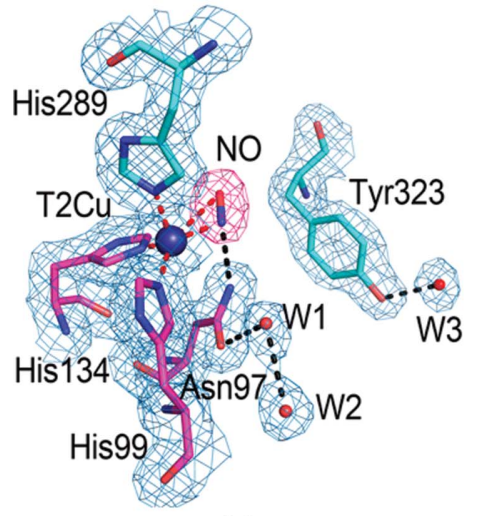

(a)

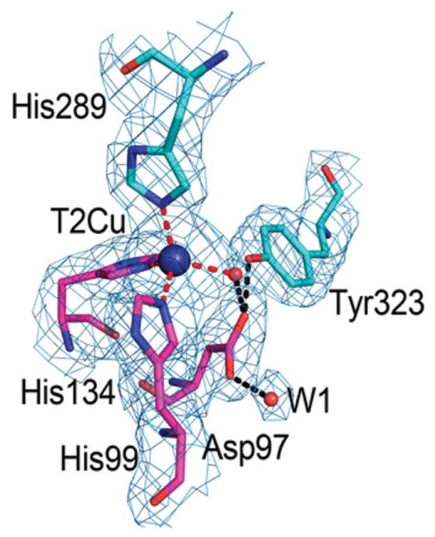

(c)

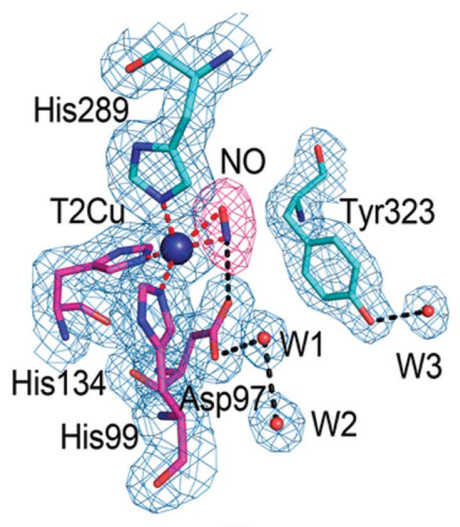

(b)

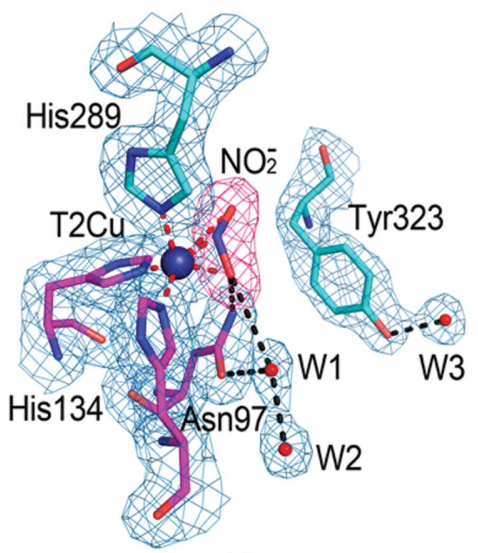

(d)
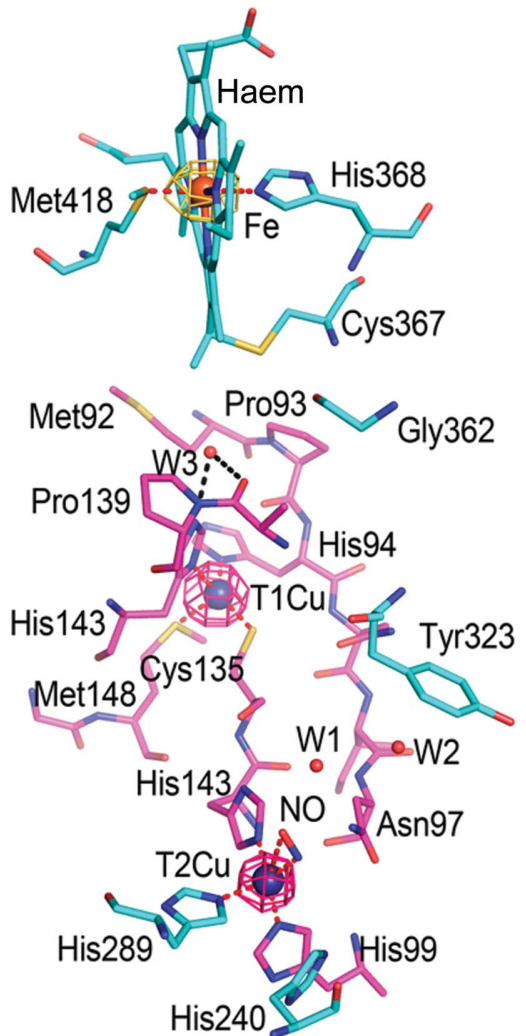

(e)

Figure 1

Details of the T2Cu sites of $R p \mathrm{NiR}$ and its D97N mutant. (a) $R p \mathrm{NiR} \mathrm{D} 97 \mathrm{~N}-\mathrm{NO}$, (b) wt $R p \mathrm{NiR}-\mathrm{NO}$ and $(c)$ wt $R p$ NiR without NO treatment in the same space group 123 . In both NO-bound structures Tyr323 is rotated away from the T2Cu site compared with the wild-type structure, where it hydrogenbonds to an aspartic acid residue. W3 is not visible in the wild type here owing to limited resolution, but is well defined in high-resolution structures. $(d)$ $R p \mathrm{NiR}-\mathrm{NO}_{2}^{-}$shows nitrite bound to T2Cu in an outward-facing manner. In $(a)$ to $(d) 2 F_{\mathrm{o}}-F_{\mathrm{c}}$ electron-density maps are shown at $1.0 \sigma$ (blue mesh) and the $F_{\mathrm{o}}-F_{\mathrm{c}}$ OMIT maps of $\mathrm{NO}_{2}^{-}$and $\mathrm{NO}$ are at $7 \sigma$ (red mesh). His 240 has been omitted for clarity. $(e)$ An $F_{\mathrm{o}}-F_{\mathrm{c}}$ OMIT anomalous map is shown at the $15 \sigma$ level around copper (1.33 $\AA$ X-ray wavelength; red mesh) and at the $20 \sigma$ level around iron (1.7 $\AA$ X-ray wavelength; orange mesh). Hydrogen bonds and copper-coordination bonds are shown as dashed black and red lines, respectively. The two adjacent monomers that form the catalytic centre are coloured magenta and cyan, respectively. 
the tyrosine is in the locked-down position protecting/blocking access to the catalytic copper.

\subsection{The structure of a substrate-bound haem CuNiR and} opening of the substrate-access channel

We obtained the first substrate-complex structure of $R p \mathrm{NiR}$ at $1.89 \AA$ resolution when crystals of $R p \mathrm{NiR} \mathrm{D} 97 \mathrm{~N}$ were

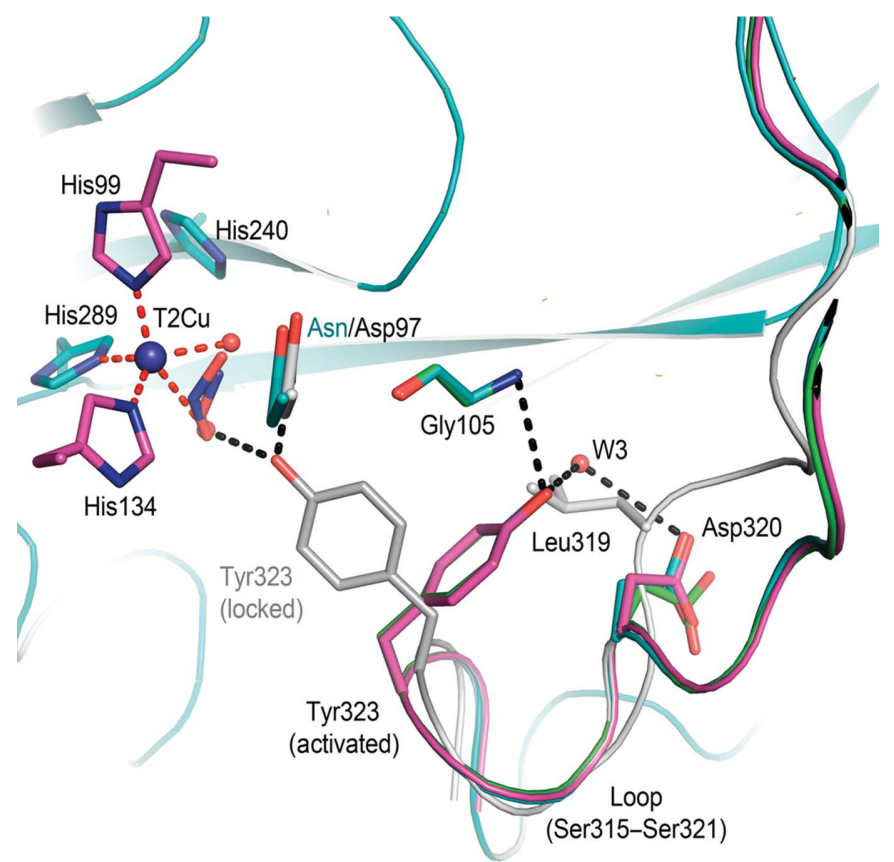

(a)

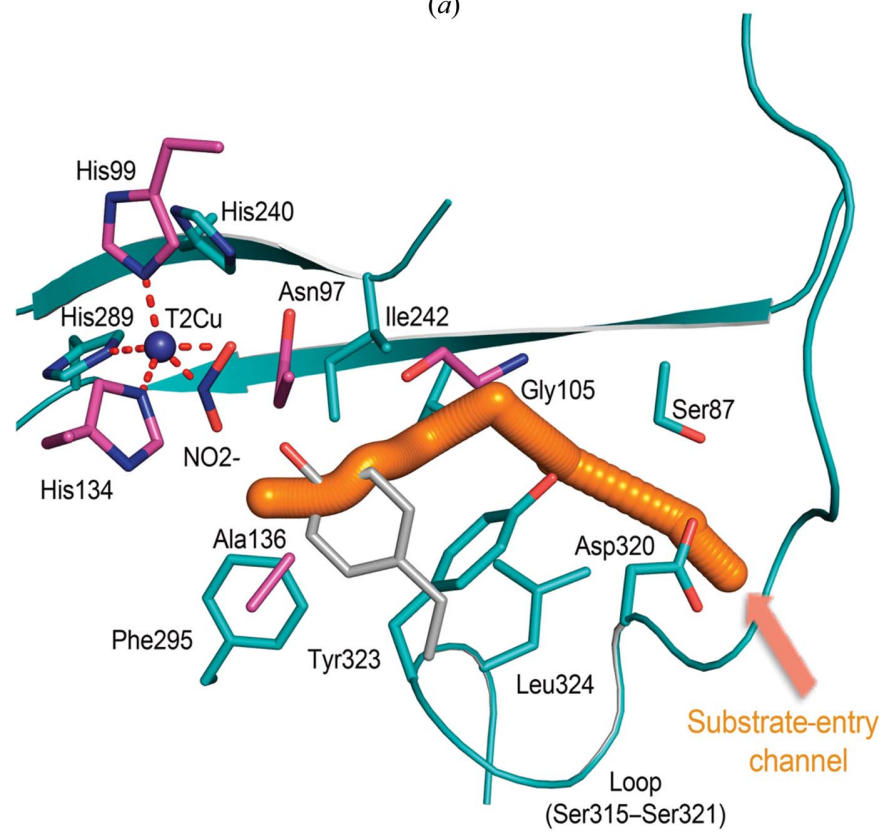

(b)

Figure 2

Details of the structural rearrangement accompanying the Tyr323 flip. (a) The wt $R p \mathrm{NiR}-\mathrm{NO}$ (green), $R p \mathrm{NiR} \mathrm{D} 97 \mathrm{~N}-\mathrm{NO}$ (cyan) and $R p \mathrm{NiR}$ $\mathrm{D} 97 \mathrm{~N}-\mathrm{NO}_{2}^{-}$(magenta) structures show a similar conformation of Tyr323 and loop (Ser315-Ser321) which differs from the free oxidized restingstate wt $R p N i R$ (grey). (b) The narrow putative NO-release channel opened by the conformational change, with resting-state Tyr323 shown in grey. $\mathrm{T} 2 \mathrm{Cu}$ coordination bonds are coloured red. Water molecules are shown as red spheres. pretreated with the product NO before soaking with nitrite. Diffusion of nitrite into NO-primed crystals showed it to bind to $\mathrm{T} 2 \mathrm{Cu}$ in a bidentate $\eta^{2}-N, O$ outward-facing manner, with distances from the $\mathrm{N}$ and two $\mathrm{O}$ atoms of nitrite to $\mathrm{T} 2 \mathrm{Cu}$ of $1.8,1.9$ and $3 \AA$, respectively (Fig. $1 d$ ). The N-coordination of $\mathrm{Cu}-\mathrm{NO}_{2}^{-}$that we observe here is the mode favoured by computational chemistry (Solomon et al., 2014) and has not been seen before in numerous structures of two-domain $\mathrm{CuNiRs}$, in which it is bound through both $\mathrm{O}$ atoms.

The substrate-access channel of the two-domain NiRs becomes apparent in the ligand-bound species of wt $R p \mathrm{NiR}$ and $R p$ NiR D97N (Fig. 2). The generation of this channel is a consequence of the new position of Tyr323, which for simplicity is called the 'activated tyrosine' position to distinguish it from the proximal 'locked' conformation as observed in asisolated wt $R p N i R$ (Antonyuk et al., 2013). The substrate channel is narrower compared with two-domain CuNiRs such as $A x \mathrm{NiR}$ (Kataoka et al., 2000; Hough, Eady et al., 2008) or
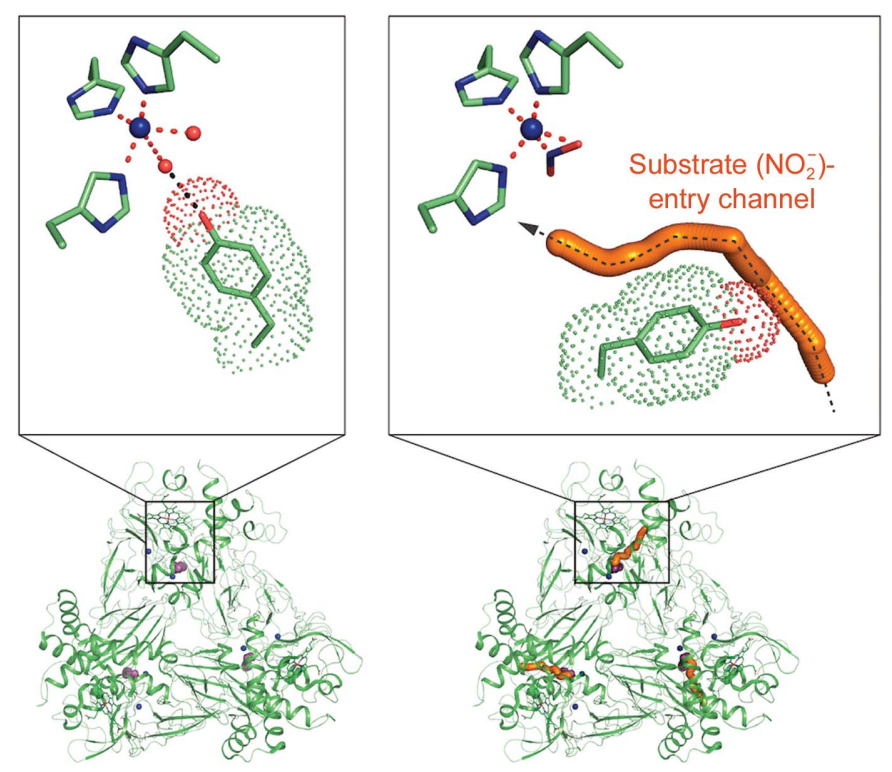

Tyrosine-activated substrate-entry channel

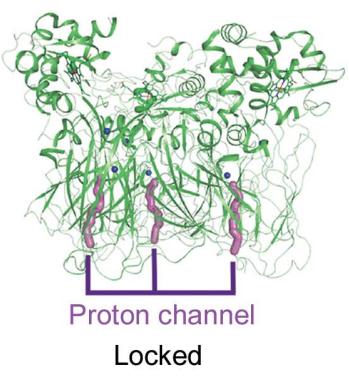

(a)

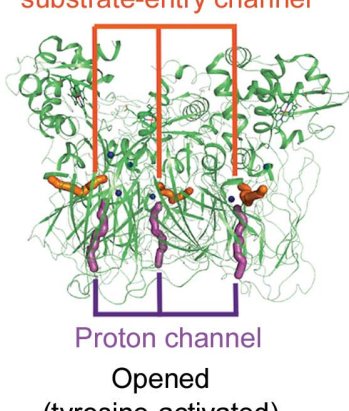

(tyrosine-activated)

(b)
Figure 3

The conformational change leading to the opening of the substrate-access channel. (a) Conformation of $R p \mathrm{NiR}$ in the resting state showing only the presence of the proton-delivery/product-release channel (magenta). (b) Activation of Tyr323 leads to opening of the substrate-access channel (orange) with the proton-delivery channel remaining unaltered. The new channel that is opened as a result of tyrosine activation is the same channel as is used for substrate access in all two-domain CuNiRs. 
AcNiR (Antonyuk et al., 2005). The substrate-binding pocket is well opened in the activated tyrosine position (Fig. 3). The channel itself is restricted by a hydrophobic filter formed by residues Tyr323, Leu324, Ile242 and Val285 that would play a significant role in controlling the passage of small molecules, including substrate. It is possible that this channel is also used by $\mathrm{NO}$ for both the activation of tyrosine and product release.

A movement of the linker loop (Ser315-Ser321) that accompanies the rotation of Tyr323 avoids a potential clash with the side chain of Leu319 (Fig. 2a). Structural reanalysis of the three-domain haem CuNiR PhNiR (PDB entry 2zoo; Tsuda et al. 2013) found that the corresponding loop (Thr305Asn311) was between the locked and activated conformations, and Tyr313 (corresponding to $R p N i R$ Tyr323) is poised to flip open without any steric hindrance (Supplementary Fig. S2). Primary-sequence analysis of different haem CuNiRs from various organisms showed that the corresponding tyrosine is highly conserved (Supplementary Fig. S1). Therefore, the channel identified here (Fig. 3) is likely to be present in all three-domain haem CuNiRs requiring the activation of tyrosine, and is used for substrate entry in a similar manner to $R p \mathrm{NiR}$ and the two-domain CuNiRs. hydrogen-bonding network of the active-site cavity or by the oxidation state of the $\mathrm{T} 2 \mathrm{Cu}$, we determined the structure of the T2Cu-deficient enzyme at $2.2 \AA$ resolution (Fig. 4a). In this structure, the side chain of Tyr323 has the same conformation as in the as-isolated wt $R p \mathrm{NiR}$ structure. However, the water that usually binds to the phenylate of Tyr 323 is absent, but the hydrogen bond $(2.6 \AA)$ to the carboxylate of Asp97 is retained. The protein has only one channel connecting the empty $\mathrm{T} 2 \mathrm{Cu}$ site to the surface of $R p \mathrm{NiR}$ on the dimer interface, similar to that in as-isolated $R p N i R$. The close similarity of $\mathrm{T} 2 \mathrm{D} R p \mathrm{NiR}$ to untreated wt $R p \mathrm{NiR}$ is clearly evident from a comparison with the atomic resolution structure of the wild-type enzyme that was obtained in the same space group (Fig. $4 b$ ).

\section{Conclusion}

Our finding that pre-treatment of crystals of $R p \mathrm{NiR} D 97 \mathrm{~N}$ with $\mathrm{NO}$ was required to open the substrate-access channel has enabled structural studies of ligand-bound species in this previously intractable system. We have identified NOmediated activation of Tyr323, a residue that is invariant in all

\subsection{Structure of reduced wt $R p \mathrm{NiR}$}

Wt $R p \mathrm{NiR}$ treated with the strong reductant dithionite showed a colour change from brown to red accompanied by a shift of the Soret band from 408 to $416 \mathrm{~nm}$, indicating reduction of the haem (Han et al., 2012). This form of reduced crystal only diffracted to a limited resolution of $\sim 4 \AA$ and the loop (315-325) containing Tyr323 was completely disordered, exposing the $\mathrm{T} 2 \mathrm{Cu}$ site. Milder reduction of wt $R p \mathrm{NiR}$ crystals by hydroxylamine or ascorbate also resulted in a change of colour, but no structural changes were observed compared with the oxidized structure except for the partial loss of the second water that is linked to Tyr323. In these cases, tyrosine remains in a locked-down position. The addition of NO to solutions of wt $R p \mathrm{NiR}$ and the D97N mutant showed no change in the optical spectrum of the haem. Thus, the activation of Tyr323 by NO does not involve reduction or binding of $\mathrm{NO}$ to haem. These observations are also consistent with the observation that mild reduction of the enzyme/haem does not activate tyrosine or cause opening of the substrate-access channel.

\subsection{Structure of T2D RpNiR}

To determine whether the locked-down conformation of Tyr323 is stabilized by the

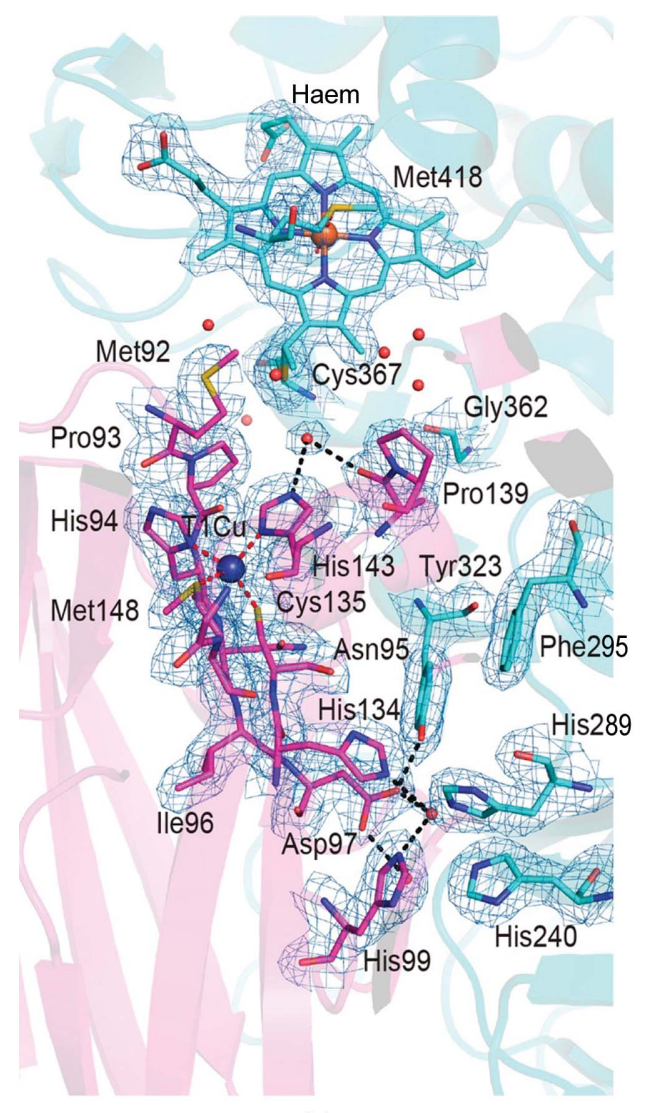

(a)

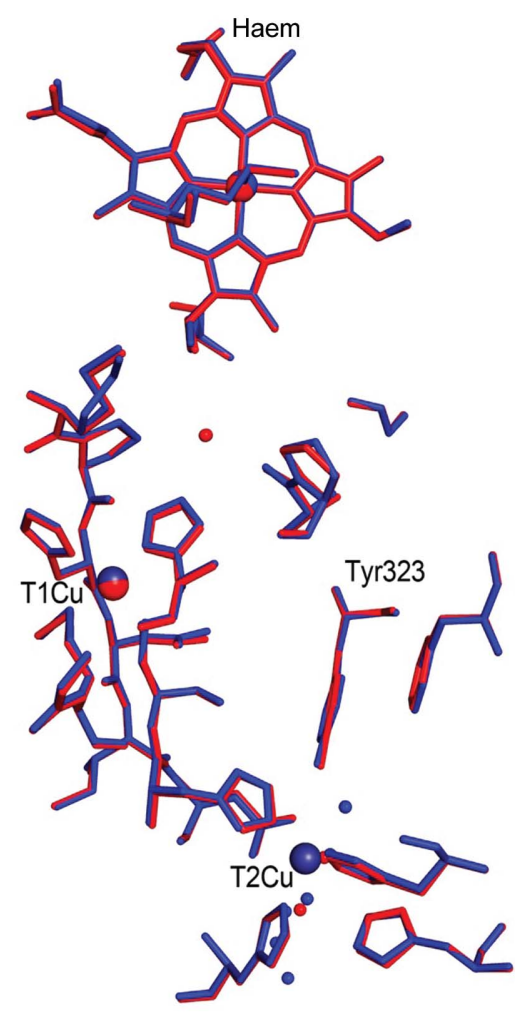

(b)
Figure 4

Cytochrome and copper-binding domains in T2D RpNiR and comparison with the fully copperloaded structure. (a) T2D $R p N i R$ has no copper in the $\mathrm{T} 2 \mathrm{Cu}$ site. Tyr323 has remained ligated to Asp97 and water and is in the locked position. $2 F_{\mathrm{o}}-F_{\mathrm{c}}$ electron-density map is shown at $1.0 \sigma$ (blue mesh). (b) Alignment of the T2D $R p N i R$ structure (red) with the untreated wt $R p N i R$ atomic resolution structure (blue; PDB entry 3ziy) showing close structural similarities, with Tyr323 in an identical position in T2D $R p N i R$ despite the absence of $\mathrm{T} 2 \mathrm{Cu}$. 

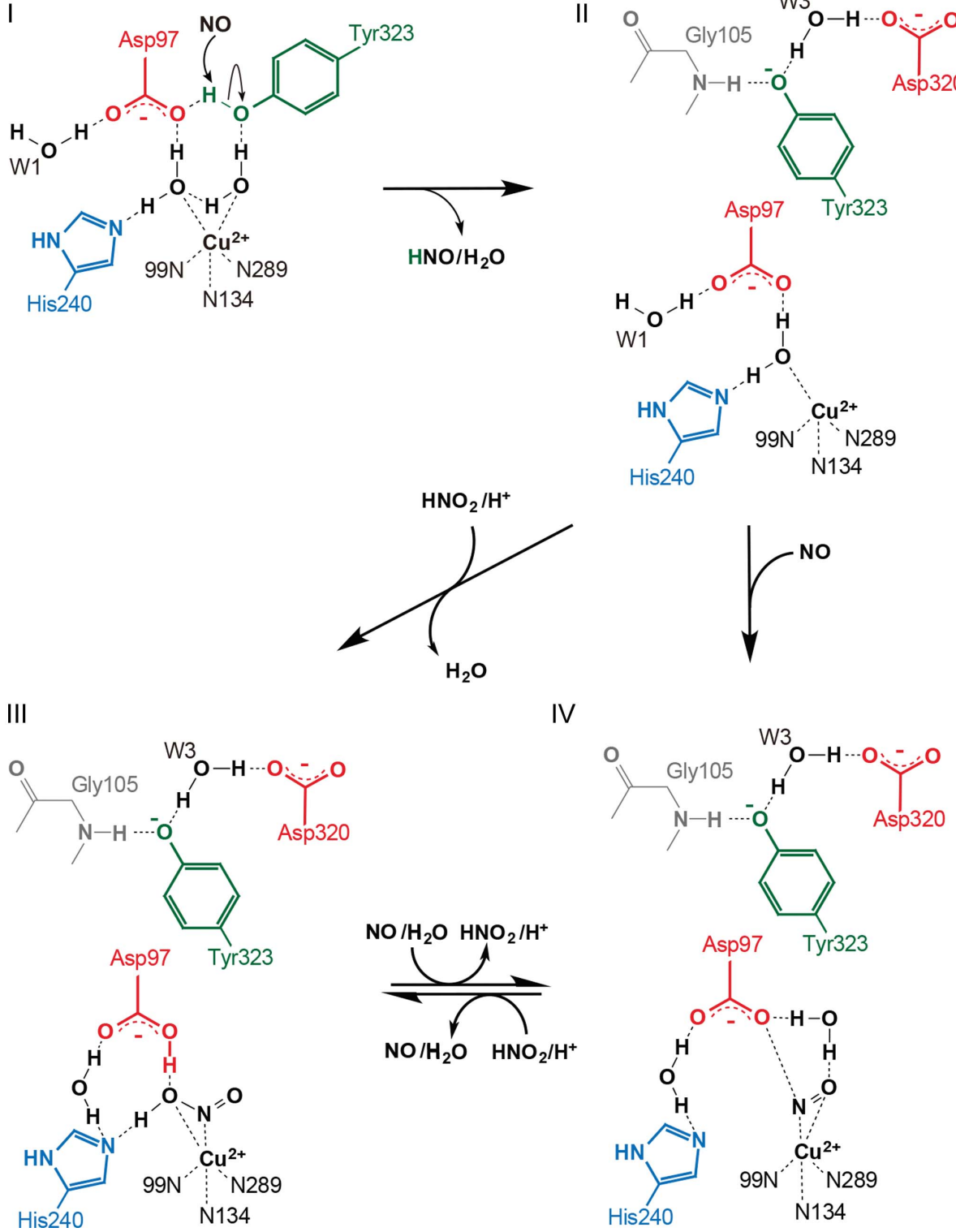

IV

Figure 5

Tyrosine-activation and substrate-binding mechanism of $R p N i R$. The conformation of Tyr 323 is changed by activation with NO by the removal of a water to make hydrogen bonds to a water (W3 hydrogen-bonded to Asp320) and the main-chain N atom of Gly105 (I to II). A substrate $\left(\mathrm{NO}_{2}^{-}\right)$ binds to the centre with the removal of a water (II to III). NO binds to the centre of the tyrosineactivated state (II) (II to IV). The formation of the substrate-binding state (III) and the NO-binding state (IV) is reversible. The $\mathrm{N}$ atoms of His 99 , His134 and His289, which are coordinated to T2Cu, are labelled 99N, N134 and N289, respectively.

haem CuNiRs, as a prerequisite to promote this structural change to prime the $\mathrm{T} 2 \mathrm{Cu}$ site for ligand binding at the catalytic centre. Both NO- and $\mathrm{NO}_{2}^{-}$-bound structures of $R p$ NiR D97N revealed rotation of Tyr323 with an accompanying water molecule, and the Ser315-Ser321 loop adopting an open conformation. These movements, which must take place prior to binding of these ligands at $\mathrm{T} 2 \mathrm{Cu}$, would result in a catalytic site that is indistinguishable from the oxidized twodomain NiRs to which nitrite binds with high affinity (Supplementary Fig. S3). The precise mechanism by which nitric oxide, which has a potential of $-0.8 \mathrm{~V}$ (Bartberger et al.,
2002), activates Tyr323 remains an open question, but what is clear is that it is a design feature of these enzymes that determines substrate entry and the availability of the catalytic site allowing substrate binding. However, our data clearly provide evidence that activation does not involve haem or catalytic copper. We propose that NO disrupts the hydrogen-bonding network around the catalytic site by capturing a proton from Tyr323 in a proton-coupled nucleophilic addition reaction to form HNO and a tyrosine radical, as observed in chemical model systems (Suarez et al., 2015). This results in the loss of the water bridging Tyr323 with copper, allowing Tyr323 to rotate away from the substrate-binding pocket (Fig. 5) together with the bridging water, producing a typical catalytic type $2 \mathrm{Cu}^{2+}$ for ligand binding. In all of the ligand-bound structures water remains associated with Tyr323. The observation that the $\mathrm{NO}_{2}^{-}$-bound structure could be obtained by soaking $R p \mathrm{NiR}$ D97N-NO crystals with sodium nitrite suggests a mechanism for substrate binding in which nitrite displaces nitric oxide (step IV to step III in Fig. 5). A corollary of this would be that once the enzyme is activated, Tyr323 remains in the activated conformation for subsequent turnover just like a switch in the open position.

The Tyr323 residue is linked to the haem centre in the cytochrome domain via three intervening residues, with the Cys364 residue tethering the haem to the domain. In the as-isolated structures two electron-transfer routes appear to be feasible: a through-bond electrontransfer route that leads to Tyr323 via Gly362 and a water-mediated electron transfer to T1Cu via His143 (Fig. 6). In the ligand-bound structures the through-bond contacts to Tyr323 are disrupted, leaving the water-mediated electron-transfer route to $\mathrm{T} 1 \mathrm{Cu}$ intact, providing evidence for the function of cytochrome in delivering electrons to the $\mathrm{T} 1 \mathrm{Cu}$ site. We propose that $\mathrm{T} 1 \mathrm{Cu}$ and $\mathrm{T} 2 \mathrm{Cu}$ are coupled in a manner similar to that established for two-domain NiRs, in which a gated mechanism delivers electron transfer to the catalytic copper when substrate nitrite binds (Brenner et al., 2009; Ghosh et al., 2009; Solomon et al., 2014; Hough, Antonyuk et al., 2008). The role of the throughbond electron-transfer route connecting Tyr323 via Gly362 is uncertain but may be involved in the latter stages of reaction. 


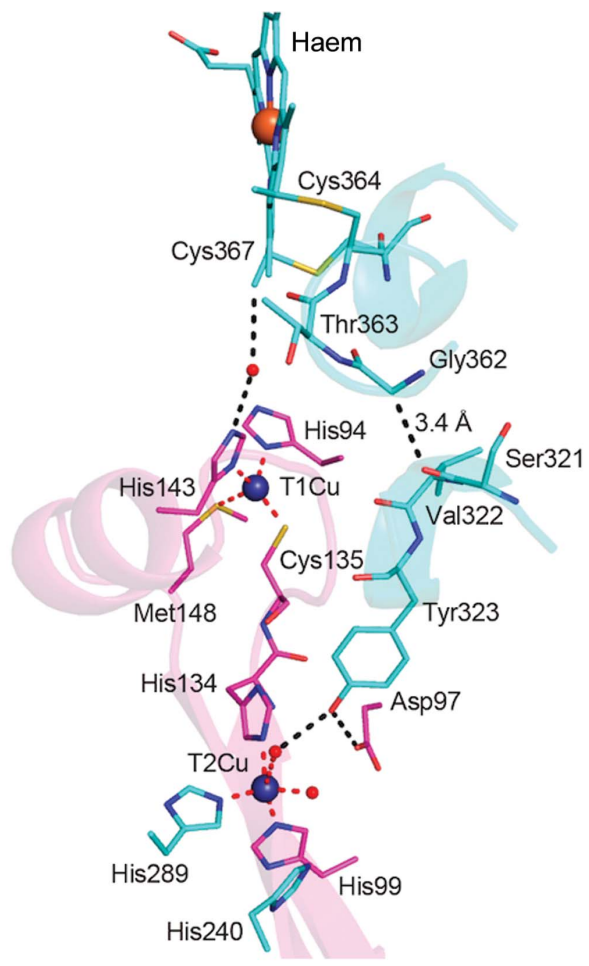

(a)

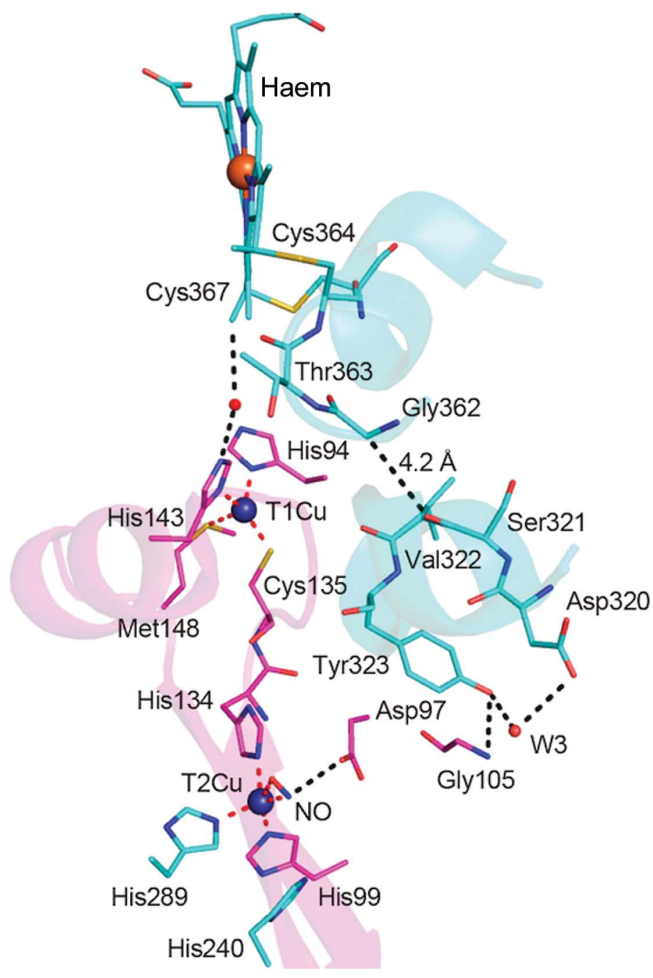

(b)

Figure 6

Possible electron-transfer routes from cytochrome to copper centres. (a) Wt $R p N i R$ and (b) $R p \mathrm{NiR}-\mathrm{NO}$. The two adjacent monomers that form the catalytic centre are coloured magenta and cyan. $\mathrm{Cu}$ and $\mathrm{Fe}$ atoms are shown as blue and orange spheres, respectively, and water molecules as red small spheres. In the wt $R p N i R$ structures two electron-transfer routes appear to be feasible: a through-bond electron-transfer route that leads to Tyr323 via Gly362 and water-mediated electron transfer to T1Cu via His143. In the ligand-bound structures the water-mediated electrontransfer route to $\mathrm{T} 1 \mathrm{Cu}$ remains.
The functional significance of the fused cytochrome domain in these enzymes has attracted some debate (Antonyuk et al., 2015). It has been suggested that the additional haem domain of $P h \mathrm{NiR}$ might not engage in direct electron transfer to the catalytic core but may have additional or different roles in controlling the specificity towards alternative putative cognate electron-donor proteins (Tsuda et al., 2013). The data presented here suggest that it does have two roles: firstly the protection of the catalytic $\mathrm{T} 2 \mathrm{Cu}$ by Tyr323 that forms part of the linker between the cytochrome and cupredoxin domains and secondly providing electrons to the $\mathrm{T} 1 \mathrm{Cu}$ centre for electron-tranfer-gated substrate reduction. The highly conserved nature of tyrosine in 13 haem CuNiRs from various organisms gains further significance from our findings and adds to the widening catalogue of roles that tyrosine plays in biological catalysis (Warren, Ener et al., 2012; Warren, Winkler et al., 2012; Glover et al., 2014; Suga, 2017). The use of tyrosine activation for the opening of the substrate channel and priming of the substrate-binding pocket may have wider implications for its use for the control and regulation of substrate binding. The involvement of NO in activating tyrosine itself through proton abstraction is intriguing. The use of tyrosine in protecting the catalytic site and its use as a switch in these enzymes is a clear example of the use of tyrosine in controlling/regulating catalysis.

\section{Acknowledgements}

We thank the staff and management of Diamond Light Source for the provision of crystallographic facilities. We would like to thank the current and past members of the Molecular Biophysics group who have contributed to the denitrification research. Our particular thanks go to Dr Mark Ellis and Dr Han Cong who worked on the $R p N i R$ programme during 2005-2008 and 2009-2012, respectively. Author contributions: RRE, SVA and SSH conceived and designed the project; JD and SVA performed the experiments; JD, DS and SVA analysed the data; JD, RRE, SVA and SSH wrote the paper.

\section{Funding information}

This work was supported by the Biotechnology and Biological Sciences Research Council, UK (grant No. BB/L006960/1 to SSH, SVA and RRE).

\section{References}

Antonyuk, S. V., Eady, R. R. \& Hasnain, S. S. (2015). In Encyclopedia of Inorganic and Bioinorganic Chemistry, edited by R. A. Scott. New York: Wiley. https://doi.org/10.1002/9781119951438.eibc2316.

Antonyuk, S. V., Han, C., Eady, R. R. \& Hasnain, S. S. (2013). Nature, 496, 123-126.

Antonyuk, S. V., Strange, R. W., Sawers, G., Eady, R. R. \& Hasnain, S. S. (2005). Proc. Natl Acad. Sci. USA, 102, 12041-12046.

Bartberger, M. D., Liu, W., Ford, E., Miranda, K. M., Switzer, C., Fukuto, J. M., Farmer, P. J., Wink, D. A. \& Houk, K. N. (2002). Proc. Natl Acad. Sci. USA, 99, 10958-10963.

Battye, T. G. G., Kontogiannis, L., Johnson, O., Powell, H. R. \& Leslie, A. G. W. (2011). Acta Cryst. D67, 271-281.

Bertini, I., Cavallaro, G. \& Rosato, A. (2006). Chem. Rev. 106, 90115. 
Boulanger, M. J., Kukimoto, M., Nishiyama, M., Horinouchi, S. \& Murphy, M. E. P. (2000). J. Biol. Chem. 275, 23957-23964.

Boulanger, M. J. \& Murphy, M. E. P. (2002). J. Mol. Biol. 315, 11111127.

Boulanger, M. J. \& Murphy, M. E. P. (2003). Protein Sci. 12, 248-256.

Brenner, S., Heyes, D. J., Hay, S., Hough, M. A., Eady, R. R., Hasnain, S. S. \& Scrutton, N. S. (2009). J. Biol. Chem. 284, 25973-25983.

Chen, V. B., Arendall, W. B., Headd, J. J., Keedy, D. A., Immormino, R. M., Kapral, G. J., Murray, L. W., Richardson, J. S. \& Richardson, D. C. (2010). Acta Cryst. D66, 12-21.

Ellis, M. J., Dodd, F. E., Sawers, G., Eady, R. R. \& Hasnain, S. S. (2003). J. Mol. Biol. 328, 429-438.

Ellis, M. J., Grossmann, J. G., Eady, R. R. \& Hasnain, S. S. (2007). J. Biol. Inorg. Chem. 12, 1119-1127.

Ellis, M. J., Prudêncio, M., Dodd, F. E., Strange, R. W., Sawers, G., Eady, R. R. \& Hasnain, S. S. (2002). J. Mol. Biol. 316, 51-64.

Emsley, P. \& Cowtan, K. (2004). Acta Cryst. D60, 2126-2132.

Evans, P. R. \& Murshudov, G. N. (2013). Acta Cryst. D69, 1204-1214.

Fukuda, Y., Tse, K. M., Lintuluoto, M., Fukunishi, Y., Mizohata, E., Matsumura, H., Takami, H., Nojiri, M. \& Inoue, T. (2014). J. Biochem. 155, 123-135.

Ghosh, S., Dey, A., Sun, Y., Scholes, C. P. \& Solomon, E. I. (2009). J. Am. Chem. Soc. 131, 277-288.

Glover, S. D., Jorge, C., Liang, L., Valentine, K. G., Hammarström, L. \& Tommos, C. (2014). J. Am. Chem. Soc. 136, 14039-14051.

Godden, J. W., Turley, S., Teller, D. C., Adman, E. T., Liu, M. Y., Payne, W. J. \& LeGall, J. (1991). Science, 253, 438-442.

Han, C., Wright, G. S., Fisher, K., Rigby, S. E., Eady, R. R. \& Hasnain, S. S. (2012). Biochem. J. 444, 219-226.

Hough, M. A., Antonyuk, S. V., Strange, R. W., Eady, R. R. \& Hasnain, S. S. (2008). J. Mol. Biol. 378, 353-361.

Hough, M. A., Eady, R. R. \& Hasnain, S. S. (2008). Biochemistry, 47, 13547-13553.

Kabsch, W. (2010). Acta Cryst. D66, 125-132.

Kataoka, K., Furusawa, H., Takagi, K., Yamaguchi, K. \& Suzuki, S. (2000). J. Biochem. 127, 345-350.

Lawton, T. J., Bowen, K. E., Sayavedra-Soto, L. A., Arp, D. J. \& Rosenzweig, A. C. (2013). J. Biol. Chem. 288, 25575-25583.
Leferink, N. G., Antonyuk, S. V., Houwman, J. A., Scrutton, N. S., Eady, R. R. \& Hasnain, S. S. (2014). Nat. Commun. 5, 4395 .

Leferink, N. G., Han, C., Antonyuk, S. V., Heyes, D. J., Rigby, S. E., Hough, M. A., Eady, R. R., Scrutton, N. S. \& Hasnain, S. S. (2011). Biochemistry, 50, 4121-4131.

Liu, J., Chakraborty, S., Hosseinzadeh, P., Yu, Y., Tian, S., Petrik, I., Bhagi, A. \& Lu, Y. (2014). Chem. Rev. 114, 4366-4469.

Maia, L. B. \& Moura, J. J. G. (2014). Chem. Rev. 114, 5273-5357.

Murshudov, G. N., Skubák, P., Lebedev, A. A., Pannu, N. S., Steiner, R. A., Nicholls, R. A., Winn, M. D., Long, F. \& Vagin, A. A. (2011). Acta Cryst. D67, 355-367.

Nojiri, M. Koteishi, H., Nakagami, T., Kobayashi, K., Inoue, T., Yamaguchi, K. \& Suzuki, S. (2009). Nature, 462, 117-120.

Prudêncio, M., Eady, R. R. \& Sawers, G. (2001). Biochem. J. 353, 259 266.

Sehnal, D., Svobodová Vařeková, R., Berka, K., Pravda, L., Navrátilová, V., Banáš, P., Ionescu, C. M., Otyepka, M. \& Koča, J. (2013). J. Cheminform. 5, 39.

Solomon, E. I., Heppner, D. E., Johnston, E. M., Ginsbach, J. W., Cirera, J., Qayyum, M., Kieber-Emmons, M. T., Kjaergaard, C. H., Hadt, R. G. \& Tian, L. (2014). Chem. Rev. 114, 36593853 .

Suarez, S. A., Neuman, N. I., Muñoz, M., Álvarez, L., Bikiel, D. E., Brondino, C. D., Ivanović-Burmazović, I., Miljkovic, J. L., Filipovic, M. R., Martí, M. A. \& Doctorovich, F. (2015). J. Am. Chem. Soc. 137, 4720-4727.

Suga, M. et al. (2017). Nature, 543, 131-135.

Tocheva, E. I., Rosell, F. I., Mauk, A. G. \& Murphy, M. E. P. (2004). Science, 304, 867-870.

Tsuda, A., Ishikawa, R., Koteishi, H., Tange, K., Fukuda, Y., Kobayashi, K., Inoue, T. \& Nojiri, M. (2013). J. Biochem. 154, 51-60.

Warren, J. J., Ener, M. E., Vlcek, A., Winkler, J. R. \& Gray, H. B. (2012). Coord. Chem. Rev. 256, 2478-2487.

Warren, J. J., Winkler, J. R. \& Gray, H. B. (2012). FEBS Lett. 586, 596602.

Winn, M. D. et al. (2011). Acta Cryst. D67, 235-242. 